\title{
Curvas de Custo para Auxiliar na Estimativa de Investimento de Capital de Processos Químicos Industriais
}

\author{
Ana C. O. Sardou, Felipe R. Moura, Ariel A. B. Berti, Victoria E. N. Santos \& \\ João B. V. Bastos
}

Devido à ascensão de novos processos na indústria química, torna-se necessário adotar ferramentas que auxiliem nas tomadas de decisão de investimentos. Muitos processos ainda não possuem estudos detalhados de curvas de custo de investimento que possibilitem uma estimativa inicial com boa precisão para diferentes capacidades de produção. Este artigo apresenta as curvas de custo e os fatores de escala para processos de produção de PET, PTA e MEG, utilizando simulação computacional como principal ferramenta. Os fatores calculados $(0,672,0,780$ e 0,784 , respectivamente) mostram como o valor tipicamente utilizado na literatura $(0,6)$ pode apresentar discrepâncias neste tipo de análise.

Palavras-chave: curva de custo; estimativa de custos de investimento; processos químicos.

Due to the increase of new processes in the chemical industry, it is necessary to adopt tools that help in investments decision-making. Many processes do not have detailed studies of investment cost curves that allows an initial estimative with good precision for different production capacities. This article presents cost curves and scale factors calculated for PET, PTA and MEG production processes using computational simulation as a main tool. The calculated factors $(0.672,0.780$ and 0.784 , respectively) show that the value typically used in the literature (0.6) can present discrepancies in this type of analysis.

Keywords: cost curve; investment cost estimate; chemical processes. 


\section{Introdução}

Para garantir que um projeto será rentável, é necessário ter uma ideia inicial do custo de produção e do investimento. Há grande dificuldade em realizar estas estimativas iniciais quando há escassez de tempo ou de uma base de dados consistente para buscar informações sem a necessidade de uma análise mais robusta. Com a ascensão de novos processos e tecnologias na indústria química, torna-se necessário criar uma metodologia que agilize os estudos das estimativas de custos de projetos e, assim, avaliar a necessidade de mudanças ou otimização no design da planta.

Existem diversos métodos que podem ser aplicados para estimar os custos de uma planta industrial. Os mais comuns são os que se utilizam de informações históricas formando bases para estimativas mais precisas, como, por exemplo, o método baseado na opinião especializada, o método da estimativa paramétrica e o método da estimativa por analogia.

O método baseado na opinião especializada é realizado or meio do método direto, normalmente sem a utilização de dados técnicos detalhados (como desenhos, listas de materiais, especificações etc.). Um profissional capacitado é ouvido e, a partir de dados e experiências em projetos anteriores, decisões são tomadas com o intuito de realizar estimativas consistentes para o projeto em questão. Como é baseado na experiência, habilidade e intuição de um profissional capacitado, o nível de detalhe do trabalho a ser realizado depende da definição técnica e do tempo designado para a execução da estimativa de custos. Esta técnica possibilita maior rapidez na elaboração da estimativa e, consequentemente, envolve menor custo, mas em contrapartida, não é respaldada por documentos técnicos (Barbosa, 2009) ${ }^{1}$.

O método da estimativa paramétrica (parametric estimate method) utiliza o procedimento de estimar os custos or meio de equações ou modelos matemáticos para relacionar uma ou mais variáveis ou características de desempenho e parâmetros físicos, tais como tamanho, volume, área, peso, potência ou consumo de energia, e associá-las ao item que está sendo estimado. Para a construção do modelo, são coletadas informações históricas de equipamentos ou construções semelhantes ao objeto de estudo que se deseja estimar e, a partir de técnicas matemáticas e estatísticas, é criado o modelo ideal para cada caso. Uma técnica estatística muito utilizada neste método para relacionar as variáveis de interesse é a regressão linear (Barbosa, 2009) ${ }^{1}$.

$\mathrm{O}$ método da estimativa por analogia (analogous estimate method) é a forma mais simples de estimativa e deve ser empregado quando existe uma significativa similaridade entre o projeto proposto e os que fazem parte da base de dados históricos. É realizado por meio de informações sobre os custos reais de projetos anteriores, com características similares de escopo, complexidade, duração, peso, tipo, tamanho, locação, requisitos e expectativas de qualidade (Barbosa, 2009) ${ }^{1}$.

Dependendo da técnica ou da ferramenta utilizada, as estimativas por analogia também são conhecidas como analogia específica (specific analogy), custos históricos (historical costs), estimativa por comparação (estimating by comparison), análise comparativa (comparative analysis), estimativa comparativa de custos (comparative cost estimating), estimativa fatorizada (factor estimating), método do fator de capacidade (capacity factor method) e estimativa do fator principal (key factor estimating) (Barbosa, 2009) ${ }^{1}$.

Dentro do escopo deste trabalho, está a estimativa de custos baseada no método do fator de capacidade (capacity factor method), em que o custo da nova instalação é obtido por meio de uma instalação similar conhecida, mas com diferente capacidade de produção. O conceito foi aplicado originalmente em 1947 por Roger Williams Jr. para estimar custos de equipamentos individuais. Em 1950, C. H. Chilton expandiu sua aplicação para estimar custos de plantas químicas completas (Baumann, 2014)2.

Segundo Baumann $(2014)^{2}$, o conceito fundamental por trás do método é o de que o custo de instalações com tecnologias similares, mas diferentes capacidades, varia de forma não-linear. DesSa forma, a razão de custo entre as duas instalações é igual à razão de suas capacidades elevada a um fator exponencial, chamado de fator de escala, como pode ser observado na Equação 1. 


$$
\frac{C_{2}}{C_{1}}=\left(\frac{Q_{2}}{Q_{1}}\right)^{n}
$$

Em que C2 é o custo requerido da instalação proposta; C1 é o custo conhecido da instalação similar existente; Q2 é a área ou capacidade da instalação proposta; Q1 é a área ou capacidade da instalação similar existente; $\mathrm{n}$ é o fator de escala (ou de capacidade) da tecnologia específica.

Para projetos de redução ou aumento de escala de plantas industriais, é necessário aplicar um fator de escala apropriado. O estudo realizado por C. H. Chilton, em 1950, indicou um fator comum igual a 0,60 para instalações químicas convencionais e a regra ficou conhecida como "regra dos seis décimos". Porém, a utilização de um fator padrão para todos os processos pode não apresentar uma boa confiabilidade ${ }^{3-9}$.

Khatiwada et al. $(2015)^{6}$ e Bruins e Sanders (2012) utilizam em seus trabalhos envolvendo biorrefinarias o fator de 0,70 para estimar os custos de plantas de produção de etanol a partir de biomassa. O estudo realizado por van den Wall Bake et al. (2008) ${ }^{8}$ apresenta uma série de curvas de custo para produção de etanol a partir de cana-de-açúcar. Suas análises reforçam a utilização de fatores variando entre 0,70 e 0,80 para processos desta natureza.

Haldi e Whitcomb (1969) analisaram 221 coeficientes de escala de diferentes processos químicos, divididos em indústrias de fertilizantes, plásticos, gases, borracha, dessalinização, refino de petróleo, alumínio, papel e celulose, entre outros. Os resultados observados indicaram que cerca de $84 \%$ dos 221 processos analisados apresentaram coeficientes menores do que 0,90. Desses, apenas $30 \%$ estavam entre 0,50 e 0,69 . Os demais apresentaram coeficientes iguais ou maiores do que 0,90 , incluindo valores acima de 1,0. O coeficiente de escala médio para estas plantas foi de 0,73 .

Segundo Towler e Sinnott $(2008)^{3}$, o expoente n é tipicamente de 0,80 a 0,90 para processos que utilizam muito trabalho mecânico ou compressão de gás. Para processos petroquímicos típicos, $\mathrm{n}$ é geralmente em torno de 0,70 . Em pequena escala, $\mathrm{n}$ de processos muito instrumentados está na faixa de 0,40 a 0,50 . Dessa forma, o valor padrão aproximado de 0,60 deve ser usado apenas quando não houver dados suficientes disponíveis para o cálculo em um processo específico.

Estes resultados só reforçam a importância de se realizarem análises que melhorem a predição de coeficientes de escala para diferentes processos e evitem a utilização do coeficiente padrão de 0,60 . Sabendo disso, outros estudos recentes foram realizados para determinar qual seria o coeficiente ideal para casos específicos, tanto para equipamentos individuais, como para instalações industriais completas.

Algumas fontes de pesquisa para fatores de escala de equipamentos individuais e instalações completas são as publicações "Process Plant Estimating Evaluation and Control", de Kenneth M. Guthrie, publicado em 1974, "Quality Guidelines for Energy System Studies, Capital Cost Scaling Methodology", publicado em 2013 pelo Departamento de Energia dos Estados Unidos (U.S. Department of Energy), e o IHS Chemical Process Economics Program.

Para estimar um fator de escala de forma mais acurada para uma instalação, a Equação 1 pode ser ajustada para um conjunto maior de dados de capacidades e custos relacionados. Estes dados podem ser analisados graficamente por meio de uma regressão de potência que gerará uma Equação correspondente, no formato da Equação 2. Dessa Equação, o expoente $\mathrm{n}$ representará o fator de escala para o tipo de instalação com a tecnologia especificada.

$$
C=a \cdot Q^{n}
$$

É importante considerar que neste tipo de estimativa de custos de processos, as tecnologias precisam ser similares quanto, por exemplo, ao tipo de processo, local e materiais de construção e tecnologias para controle de emissões. Também é necessário observar o intervalo de capacidades em que o 
fator de escala foi calculado. $\mathrm{O}$ fator pode ser utilizado para calcular o custo de processos em que a capacidade de produção esteja fora do intervalo considerado no escalonamento, porém com cautela pois o erro na extrapolação pode ser maior que o erro considerado no ajuste.

A realização de um estudo adequado de pré-viabilidade econômica é essencial para o bom planejamento das etapas iniciais de um projeto e utilizar o coeficiente de escala como ferramenta para auxiliar nestas estimativas pode ser um bom critério. Para isso, é importante estabelecer um padrão para o método utilizado, a fim de aumentar a confiabilidade e reduzir as incertezas destas análises de custos de processos químicos.

Dessa forma, o objetivo principal deste artigo é apresentar os coeficientes de escala de três processos tradicionais da indústria química, obtidos por meio de estimativas de custo robustas, a fim de contribuir para a literatura com dados de custo para a elaboração de outros trabalhos, em particular aqueles que visam ao estudo e à otimização de cadeia de valor.

O presente artigo está estruturado de forma a apresentar, num primeiro momento, nesta introdução, o contexto motivador do presente estudo e dos métodos usuais de estimação de custos. Na segunda parte, está descrita a metodologia utilizada para adaptar o método do fator de capacidade e aplicá-lo a diferentes plantas químicas. Na terceira seção, os três casos avaliados são detalhados, evidenciando os principais pontos de atenção de cada aplicação. Em seguida, na quarta seção, os resultados são discutidos com embasamento em estudos similares já desenvolvidos, demonstrando a efetividade da adaptação proposta no presente estudo. $\mathrm{Na}$ última seção, são apresentadas as principais conclusões e perspectivas do estudo.

\section{Metodologia}

Com o auxílio de softwares especializados que possibilitam simular e avaliar economicamente plantas industriais, foi adotada uma metodologia de construção e análise técnico-econômica de processos que é ilustrada no diagrama da Figura 1 e será explicada a seguir.

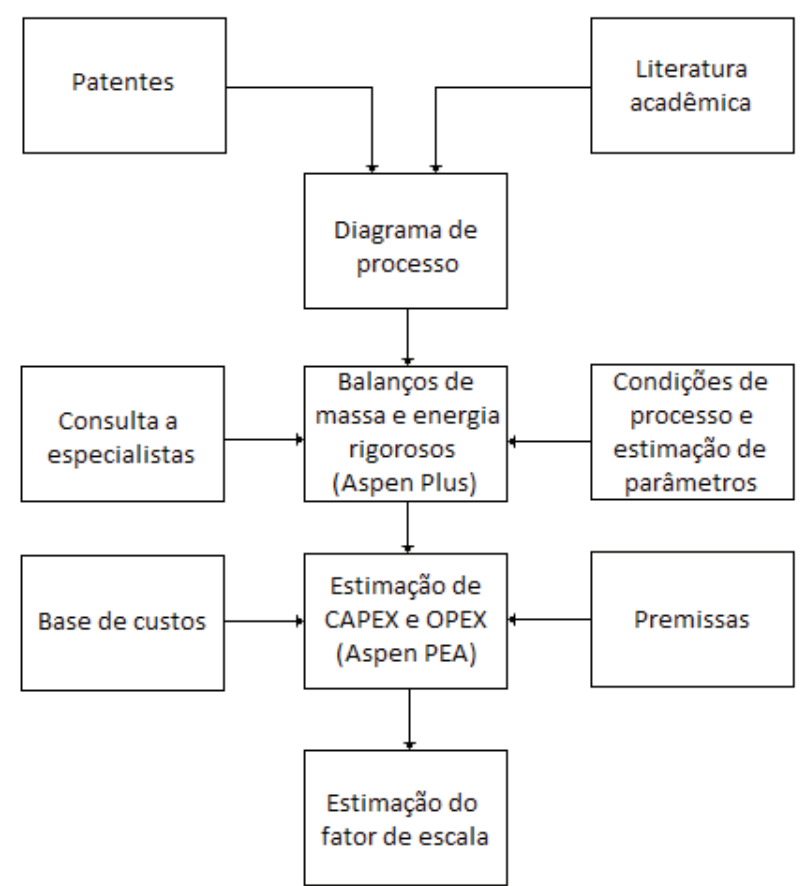

Figura 1. Fluxograma simplificado da abordagem adotada para a realização de análises técnico-econômicas de processos.

Inicialmente, foi realizado um levantamento técnico-científico de informações na literatura, tanto de patentes quanto de teses e artigos relacionados aos produtos de interesse. A partir destas pesquisas, foram identificadas as rotas disponíveis, suas vantagens e desvantagens e uma foi escolhida. Com isso, foi desenvolvido um fluxograma de processo adequado e, em seguida, uma simulação baseada neste fluxograma foi construída utilizando o software Aspen Plus ${ }^{\circledR}$. A partir desta simulação, foram obtidos os balanços de massa e energia do processo escolhido.

A análise de custos dos equipamentos foi realizada com auxílio do Aspen Process Economic Analyzer ${ }^{\circledR}$, que possui uma base própria de custos atualizada anualmente. $\mathrm{O}$ dimensionamento dos equipamentos e as estimativas de consumo de matéria-prima e utilidades foram baseados nos balanços de massa e de energia obtidos com a simulação de cenários de operação usando o modelo desenvolvido. 
As estimativas dos custos totais do projeto foram divididas em CAPEX e OPEX. As estimativas de CAPEX englobam os custos de instalação da unidade de processo, que incluem aqueles relacionados à aquisição de equipamentos e os associados à montagem da unidade. Já as estimativas de OPEX englobam os custos operacionais da unidade, que incluem os que estão relacionados ao consumo de matéria-prima e utilidades e os associados aos custos com mão-de-obra, manutenção, entre outros.

Por fim, a determinação do fator de escala foi realizada com o cálculo do CAPEX (custos de investimento) para diferentes capacidades do mesmo processo estudado, regredindo os dados de acordo com a Equação 2. As capacidades estudadas foram aumentadas ou reduzidas em $30 \%$ em relação à capacidade anterior até um limite pré-estabelecido. Cada valor de capacidade foi simulado no modelo desenvolvido no Aspen Plus ${ }^{\circledR}$ e o procedimento de análise de custos foi repetido no Aspen Process Economic Analyzer ${ }^{\circledR}$.

Com os custos de CAPEX relacionados às capacidades de produção da planta, foi criado um gráfico em Excel incluindo uma linha de tendência em formato de potência, gerando, assim, a curva de custo correspondente aos custos de investimento do processo. $\mathrm{O}$ expoente da Equação gerada corresponde ao fator de escala.

\section{Processos Estudados}

Três estudos de caso são apresentados a seguir. Os processos escolhidos para a construção das curvas de custo deste artigo foram os de produção de ácido tereftálico purificado (PTA), de politereftalato de etileno (PET) e de monoetileno glicol (MEG).

A escolha das capacidades das simulações usadas como base para as análises econômicas foi feita em função das plantas industriais já existentes atualmente. $\mathrm{O}$ Complexo PQS possui uma unidade de produção de PTA com capacidade para produzir 700 mil toneladas por ano. O Complexo também possui atualmente uma unidade de produção de PET com capacidade de 225 mil toneladas por ano e pretende aumentar esta produção para 450 mil toneladas por ano (PQS, 2018) ${ }^{9}$. A Shell possui uma unidade de produção de MEG com capacidade de 750 mil toneladas por ano (SHELL, 2018) ${ }^{10}$.

\section{PTA}

Ácido tereftálico purificado (PTA) é a matériaprima predominante para a produção de PET e cerca de $70 \%$ de sua produção a partir de p-xileno é destinada ao processo de manufatura deste polímero. O ácido tereftálico é encontrado na forma de um pó branco, porém sua estocagem é frequentemente realizada na forma fundida. Possui leve solubilidade em água e álcoois e sublima quando é aquecido. A Figura 2 apresenta o fluxograma simplificado do processo de produção do PTA a partir da oxidação de p-xileno.

A Área 100 contém as operações unitárias responsáveis pela oxidação do p-xileno, cristalização e posterior filtração dos cristais de ácido tereftálico bruto (CTA), assim como os equipamentos para o reator de tratamento de gás de exaustão e recuperação do solvente. A Área 200 é responsável por cristalizar, filtrar e secar os cristais de PTA formados no reator de hidrogenação de CTA. A Área 300 é a seção responsável por recuperar o catalisador metálico utilizado na reação de oxidação do p-xileno.

\section{PET}

Politereftalato de etileno (PET) é um polímero termoplástico amplamente utilizado para a produção de fibras sintéticas, embalagens para a indústria alimentícia e de bebidas, entre outras aplicações. Em estado sólido, é encontrado como uma resina incolor e semi-cristalina. Uma rota comum de obtenção deste polímero é a partir da esterificação do PTA, processo apresentado no fluxograma da Figura 3.

A Área 100 contém dois reatores em série para esterificação de PTA equipados com colunas para separar a água proveniente do processo e retornar o etileno glicol (EG) para os reatores. A Área 200 é composta por um reator de pré-polimerização e um posterior reator 
responsável por formar os pellets de PET. Na Área 300, os pellets são enviados para uma série de cristalizadores e o produto final é resfriado e enviado para estocagem. A Área 400 é responsável por aquecer e destilar o EG para retirar água e outros voláteis, a fim de reaproveitá-lo no início do processo.

\section{MEG}

Monoetileno glicol (MEG) é um composto orgânico amplamente utilizado na manufatura de fibras de poliéster e como anticongelante. Em sua forma pura líquida, é um composto inodoro e incolor. A principal rota atual de fabricação de MEG é a partir de eteno, tendo como intermediário o óxido de etileno (EO). Para aumentar a seletividade, no processo OMEGA da Shell é sugerido que o EO seja inicialmente convertido com dióxido de carbono (CO2) para formar carbonato de etileno (EC). A Figura 4 apresenta o fluxograma simplificado do processo utilizado para este trabalho.



Figura 2. Fluxograma simplificado do processo de produção de PTA.

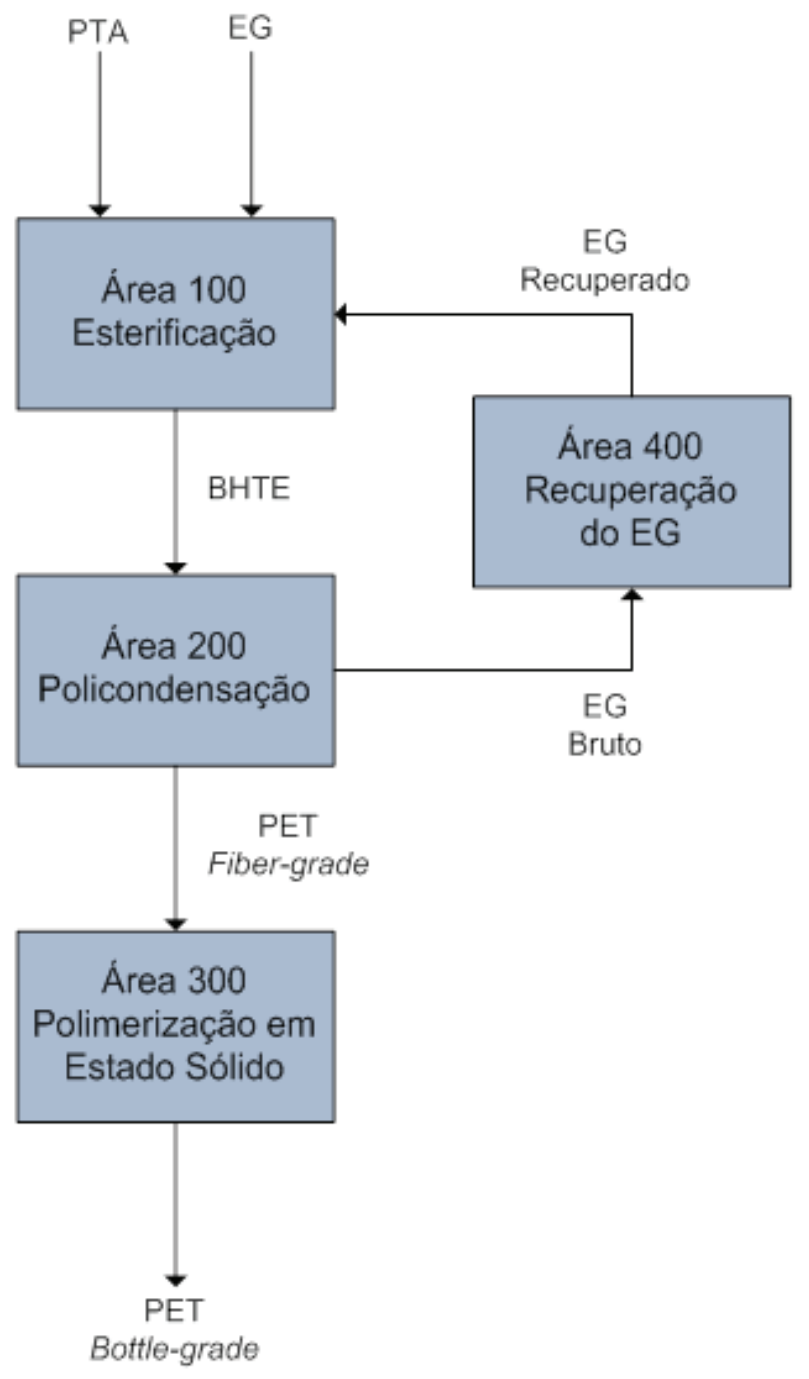

Figura 3. Fluxograma simplificado do processo de produção de PET.

A Área 100 contém um reator de oxidação de eteno para formação de EO e colunas responsáveis por recuperar o eteno não reagido e enviá-lo de volta para o início do processo. A Área 200 possui uma série de reatores responsáveis pela síntese do EC a partir de EO e CO2 na presença de catalisador. $\mathrm{Na}$ Área 300, dois reatores hidrolisam o EC para a 
formação de etileno glicol. A Área 400 contém uma coluna para purificar o etileno glicol proveniente da hidrólise do EC e uma unidade para separar o catalisador proveniente da Área 200 e retorná-lo para os reatores de síntese de EC.

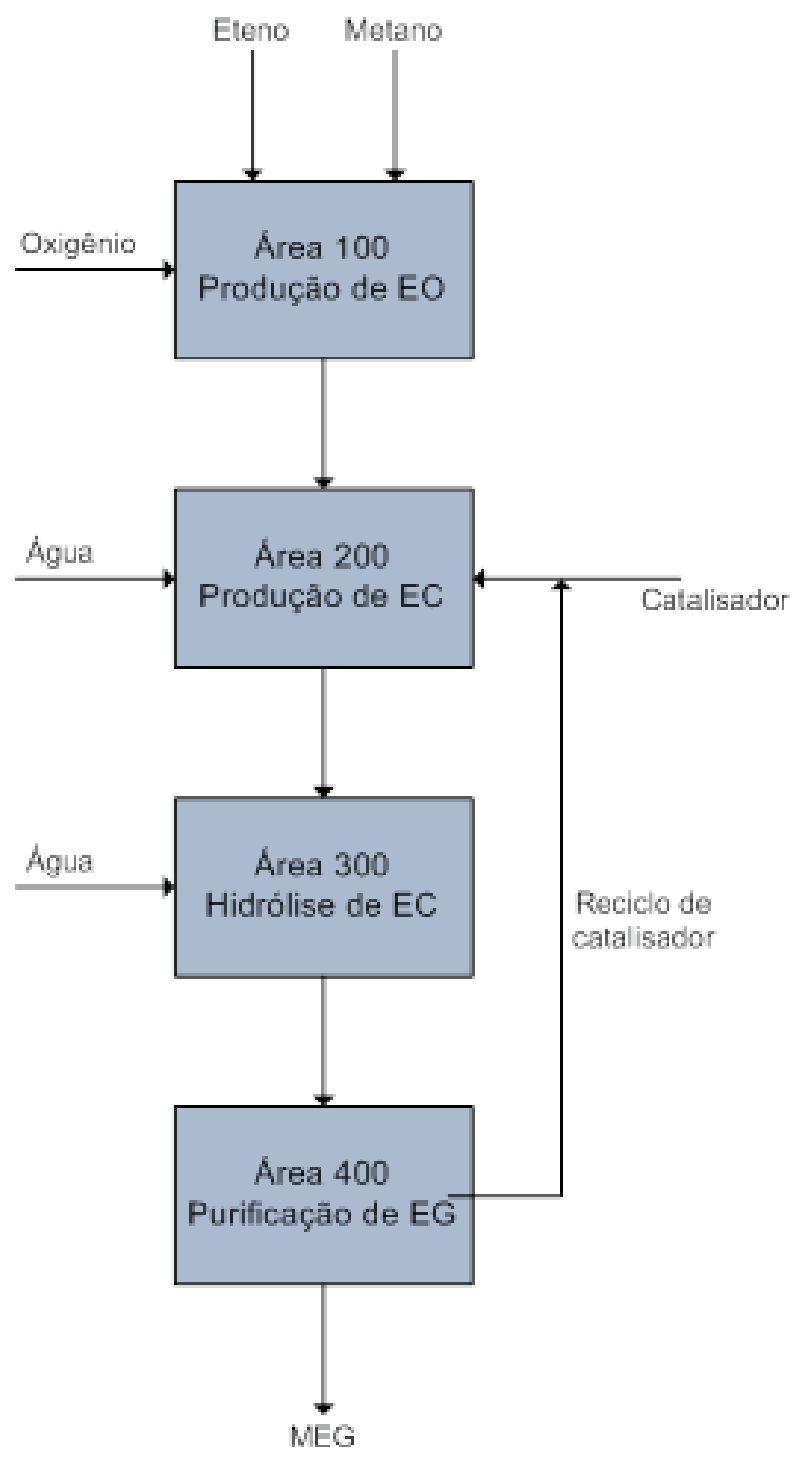

Figura 4. Fluxograma simplificado do processo de produção de MEG.

\section{Apresentação de Resultados}

Seguindo as premissas anteriormente explicadas para a escolha das capacidades de produção presentes nas curvas de custo, optou-se por realizar as análises de PTA com as capacidades variando entre 300 e 850 mil toneladas por ano. Para a análise da curva de custo de PET, as variações foram realizadas em intervalos entre 100 e 350 mil toneladas por ano. Já para a análise das plantas de MEG, o intervalo considerado foi entre 200 e 750 mil toneladas por ano. As curvas de custo resultantes das análises podem ser observadas nos Gráficos 1, 2 e 3.

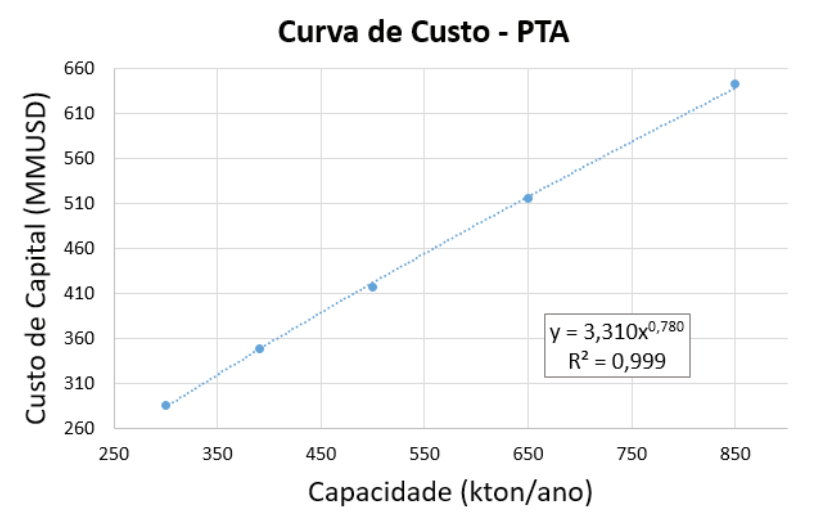

Gráfico 1. Curva de custo do processo de obtenção de ácido tereftálico purificado (PTA).

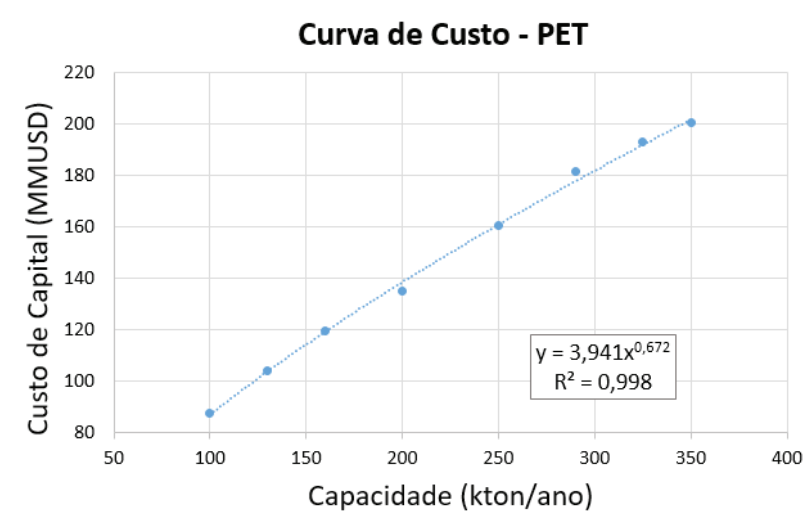

Gráfico 2. Curva de custo do processo de obtenção de politereftalato de etileno (PET). 


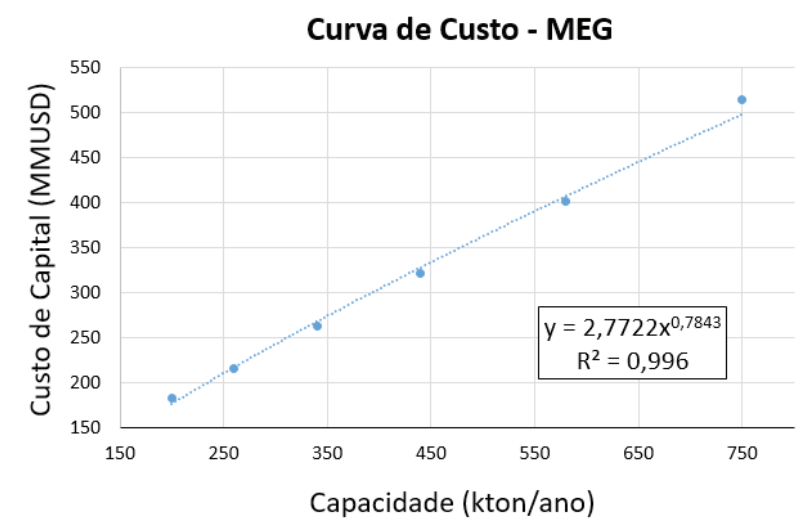

Gráfico 3. Curva de custo do processo de obtenção de monoetileno glicol (MEG).

Cada gráfico ilustra as relações obtidas entre as capacidades das plantas e os custos de capital (CAPEX). Nota-se também a presença das equações de potência características e os respectivos fatores de escala de cada processo, o que são evidenciadas pelos altos valores dos coeficientes de correlação $(0,999,0,998$ e 0,996, respectivamente). Os fatores correspondentes aos processos de PET, PTA e MEG foram, respectivamente, 0,672, 0,780 e 0,784 .

Retornando à regra dos seis décimos, é perceptível que nenhum dos processos aqui estudados apresentaria um bom resultado caso a estimativa de custos fosse realizada utilizando o fator de escala sugerido na literatura de 0,60 . O resultado do processo que se encaixaria melhor seria o de PET, mas ainda assim apresentando grandes discrepâncias. Dessa forma, as análises aqui realizadas contribuem para a formação de um banco de dados mais acurado para estimativas de custos dos processos aqui tratados.

\section{Conclusão}

O método do fator de capacidade (capacity factor method) apresenta uma boa proposta quanto à estimativa inicial de custos de projetos. Quando aplicado corretamente, o fator de escala possibilita resultados confiáveis para serem utilizados em análises futuras. A revisão da literatura referenciada demonstra a importância deste método para a estimativa de custos de capital de processos. Foi visto que, pesquisas que se propõem a realizar estudos de cadeias de valor e análises técnico-econômicas de processos utilizam este método para efetuar a estimativa inicial de seus custos, embora artigos encontrados utilizem o fator de escala de 0,60 , valor tipicamente sugerido na literatura.

A metodologia da construção das curvas de custo apresentadanesteartigo, utilizandosimulaçãocomputacional para cálculos de balanços de massa e energia e cálculos de CAPEX, se mostrou eficiente e precisa. Uma vez definido o processo e construída a simulação foi possível obter os custos de diferentes capacidades dos processos estudados de forma ágil, possibilitando a construção das curvas.

Os resultados encontrados mostram que, para os processos estudados, o valor comumente sugerido na literatura para o fator de escala não representa de forma acurada os custos do processo, causando discrepâncias quando estimativas de custo de diferentes capacidades são realizadas. A propagação de erros na estimativa inicial de um projeto acarreta em falhas nas tomadas de decisões estratégicas, podendo gerar grandes prejuízos econômicos. Portanto, uma boa forma para estimar fielmente os custos de um processo é com a utilização de um fator de escala determinado especificamente para ele. Dessa forma, este artigo contribui para a literatura com fatores de escala de processos de produção de PTA, PET e MEG.

\section{Referências}

1. Barbosa, C. Gerenciamento de Custos em Projetos. 4th ed. FGV Management, 2009. 146.

2. Baumann, C. T. Cost-to-Capacity Method: Applications and Considerations. The M\&Ts Journal: 2014.

3. Towler, G.; Sinnott, R. Chemical Engineering Design: Principles, Practice and Economics of Plant and Process Design. 1st ed. Elsevier, 2008. 1245.

4. Garrett, D. E. Chemical Engineering Economics. 1st ed. Van Nostrand Reinhold, 1989.

5. Happle, J.; Jordan, D. G. Chemical Process Economics. 2nd ed. Marcel Dekker, 1975.

6. Khatiwada, Dilip et al. Optimizing ethanol and bioelectricity 
production in sugarcane biorefineries in Brazil. Renewable Energy, n. 85, p. 371-386, jun. 2015.

7. Bruins, Marieke E.; Sanders, Johan P. M. Small-scale processing of biomass for biorefinery. Biofuels, Bioproducts \& Biorefining, n. 6, p. 135-145, fev. 2012.

8. Van Den Wall Bake, J. D. et al. Explaining the experience curve: Cost reductions of Brazilian ethanol from sugarcane. Biomass \& Bioenergy, n. 33, p. 644-658, dez. 2008.

9. <http://www.pqspe.com.br/>. Acesso em: 15 ago. 2018.

10. $<$ https://www.shell.com/business-customers/chemicals/factsheetsspeeches-and-articles/factsheets/mono-ethylene-glycol.html $>$. Acesso em: 15 ago. 2018.

\section{Ana C. O. Sardou',2*, Felipe R.} Moura',3, Ariel A. B. Berti' ${ }^{1}$, Victoria E. N. Santos ${ }^{1,3}$ \& João B. V. Bastos ${ }^{1,3}$

${ }^{1}$ Instituto SENAI de Inovação em Biossintéticos, SENAI CETIQT, CEP 20961-020, Rio de Janeiro, Brasil

${ }^{2}$ Faculdade SENAI CETIQT, CEP 20961-020, Rio de Janeiro, Brasil

${ }^{3}$ Escola de Química, Universidade Federal do Rio de Janeiro, CEP 21941-909, Rio de Janeiro, Brasil

*E-mail: carolsardou96@gmail.com 